\title{
Frontières
}

\section{Complexités et défis des deuils après suicide}

\section{Michel Hanus}

Volume 21, numéro 1, automne 2008

Prévenir le suicide

URI : https://id.erudit.org/iderudit/037869ar

DOI : https://doi.org/10.7202/037869ar

Aller au sommaire du numéro

Éditeur(s)

Université du Québec à Montréal

ISSN

1180-3479 (imprimé)

1916-0976 (numérique)

Découvrir la revue

Citer cet article

Hanus, M. (2008). Complexités et défis des deuils après suicide. Frontières, 21(1), 10-14. https://doi.org/10.7202/037869ar

\section{Résumé de l'article}

Sa propre mort est toujours une expérience troublante et mystérieuse. Celle des autres met en deuil dans la souffrance. Lorsque la mort est provoquée par le défunt lui-même, la situation est plus complexe, le deuil plus difficile. Certes tous les éléments du deuil et du travail de deuil habituels se retrouvent dans ces deuils traumatiques, mais ils comportent des aspects spécifiques : le suicide est encore considéré comme une mauvaise mort, ce qui risque de stigmatiser l'entourage. Il entraîne un état de choc et une dépression intenses. Les images traumatiques du défunt retardent le travail de deuil et torturent ceux qui les vivent. Les sentiments de culpabilité sont extrêmes. Ces deuils difficiles justifient un accompagnement approprié, surtout chez les enfants et adolescents. 


\section{Résumé}

Sa propre mort est toujours une expérience troublante et mystérieuse. Celle des autres met en deuil dans la souffrance. Lorsque la mort est provoquée par le défunt lui-même, la situation est plus complexe, le deuil plus difficile. Certes tous les éléments du deuil et du travail de deuil habituels se retrouvent dans ces deuils traumatiques, mais ils comportent des aspects spécifiques: le suicide est encore considéré comme une mauvaise mort, ce qui risque de stigmatiser l'entourage. II entraîne un état de choc et une dépression intenses. Les images traumatiques du défunt retardent le travail de deuil et torturent ceux qui les vivent. Les sentiments de culpabilité sont extrêmes. Ces deuils difficiles justifient un accompagnement approprié, surtout chez les enfants et adolescents.

Mots clés: suicide - mort deuil - dépression - culpabilité accompagnement.

\section{Abstract \\ One's own death is always a disturbing and mysterious experience. Other's bring about mourning and suffering. When death is caused by the deceased him- self, the situation is more complex, the grieving process more difficult. Of course, all the usual elements of the mourning and grieving process are found in these traumatic bereavements but they entail specific aspects: suicide is still regarded as a bad death, which may stigmatize the close ones. It leads to a state of shock and an intense depression. Traumatic images of the deceased delay the work of mourning and torture those who live it. Feelings of guilt are extreme. These difficult bereavements call for an appro- priate response, especially with children and adolescents.}

Keywords: suicide - death bereavement - depression - guilt caregiving.

$\begin{array}{llllllll}\text { A } & \mathrm{R} & \mathrm{T} & \mathrm{I} & \mathrm{C} & \mathrm{L} & \mathrm{E} & \mathrm{S}\end{array}$

\section{Complexités et défis des deuils après suicide}

\section{Michel Hanus, M. D., Ph. D., président de la Société de thanatologie, président de la Fédération européenne Vivre son deuil.}

La mort brutale du suicide entraîne un deuil traumatique particulier, difficile et habituellement très long sans qu'il soit pour autant compliqué ou pathologique bien qu'il puisse le devenir chez des personnes antérieurement fragilisées. Un accompagnement de ces personnes est nécessaire en particulier pour les enfants et adolescents. Commençons par réfléchir à cette mort particulière qu'est le suicide dont nous essayerons ensuite d'approcher les motivations. Ainsi nous arriverons à préciser les particularités de ces deuils.

\section{UNE MORT PARTICULIÈRE}

La mort est une compagne familière et fidèle que nous cherchons bien souvent à éloigner sinon même à répudier. Mais elle ne se laisse pas faire et finit toujours par avoir le dernier mot. S. Freud, déjà au printemps de 1915, écrivait que, en tant qu'individus, nous n'y croyons pas vraiment, nous la considérons comme un accident, une éventualité malheureuse mais pas vraiment comme une nécessité et, en tant que groupe social, «nous essayons de la tuer par notre silence». Elle est déjà si pénible de marquer irrévocablement notre finitude et de nous séparer irrémédiablement de ceux que nous aimons. Mais elle est tellement mystérieuse - mystère des mystères que l'humanité a si longtemps confié à la transcendance dans les religions - car la mort des autres ne nous dit que bien peu de chose de la nôtre. Dans le même texte, Freud dit que nous n'avons pas de représentation même inconsciente de notre propre mort, dans la mesure où elle constitue l'extrême du négatif, donc irreprésentable. Dans un autre texte (Freud, 1924), il dit que c'est l'inconnu qui fait le plus peur à l'être humain, tout en stimulant sa curiosité.

Nombreuses ont été et nombreuses sont encore les tentatives pour dissiper partiellement l'obscurité de la mort. Déjà les progrès initiés et largement développés maintenant du mouvement des soins palliatifs peuvent laisser espérer à chacun de ne pas mourir dans la souffrance. Mais toute souffrance est-elle éradicable? N'estelle pas aussi parfois un cri qui vient du fond de nous-mêmes et qui ne peut s'apaiser en partie que d'être entendu, compris et respecté ? Les personnes qui ont vécu des «expériences proches de la mort» et celles qui ont pu la frôler sans appréhension, sinon avec sérénité, voire avec jouissance, nous incitent à penser que le grand passage n'est pas toujours si difficile. Mais, en dernière analyse, qu'en sera-t-il pour nous? Nous n'en savons rien. Dès que les circonstances de notre vie se compliquent, dès que notre goût de vivre s'affadit, nous sentons la mort rôder autour de nous. Des personnes éminentes nous disent que «la mort n'existe pas ». Mais la mort refuse de ne pas exister et elle emporte même ceux qui la refusent ou la dénient.

La plupart des religions et des philosophies nous disent que l'existence transcende la vie et qu'après la mort existe 
une autre vie. Comme cette position est invérifiable avant notre propre mort, c'est affaire de pure croyance. La mort effracte la distance entre la profondeur de l'être et ses superficialités, abrase l'écart qui sépare l'inconscient de la conscience, affiche ce que nous sommes à l'écran de ce que nous croyions être. La religion juive mais encore plus la musulmane ont inscrit le jugement dans le moment de la mort. Que ce soit le Christ ou les anges de la mort qui, nous diton, viennent nous demander des comptes, n'est-ce pas simplement que nous sommes confrontés, dans ces ultimes moments, à l'estimation de notre conscience, ce que Freud a appelé le surmoi et l'idéal du moi? Avons-nous le sentiment d'avoir accompli, dans la mesure de nos moyens, notre vie, sommes-nous en paix avec nous-même?

Aussi bien pour nous encourager à écarter cette pénible pensée de notre conscience, s'est-on efforcé d'en donner, voire d'en imposer, une image sinon simplement négative, terrifiante. C'est la négativité absolue pour Freud, qui n'était pas vraiment à l'aise avec elle, et nous avons vu que la négativité est bien proche de l'inexistence. Pour la religion catholique, la mort, c'est le mal par excellence et le Christ ressuscité est vainqueur de la mort; d'ailleurs, nous allons tous ressusciter. Seules les religions d'Orient acceptent de penser la disparition individuelle dans le nirvana du grand tout lorsque la succession des réincarnations a amené l'être à un degré suffisant de purification et de détachement.

Ainsi la mort relativement pacifiée est-elle la mort préparée, la mort attendue, la mort consentie, une mort à visage humain en quelque sorte, une mort rassurante, exemplaire. Mais de ce que vit et ressent le mourant nous n'avons que des signes parfois trompeurs. Toujours est-il que cette vision qui se veut apaisée de la mort vole en éclats lorsque la mort est brutale, violente, traumatique, inattendue, soudaine et encore plus lorsqu'elle frappe de cette manière un être jeune, un enfant, un adolescent, un jeune adulte et on reste toujours l'enfant de ses parents, quel que soit son âge.

Mais voilà que 11000 fois par an environ en France, cette mort terrible n'est pas due aux circonstances malheureuses de quelque accident: il s'est tué, elle s'est tuée, c'est un suicide! Alors la mort est plus affreuse encore. Écrasée entre l'incrédulité, l'incapacité d'accepter et l'impossibilité de récuser la réalité des faits, la personne en deuil devient sous le coup de cette horrible nouvelle - qu'elle soit amenée à découvrir le corps ou qu'elle l'apprenne par ouï-dire - à la fois confuse, anesthésiée, comme glacée.
La mort que nous essayons, au cours de notre vie, d'apprivoiser afin de l'aborder avec plus de sérénité s'impose alors de manière effrayante. Elle est toujours inattendue même lorsqu'il a existé des tentatives de suicide ou un état dépressif intense; toutes les personnes qui font et ont fait des tentatives n'en meurent pas et tous les déprimés ne se suicident pas! Mais elle est encore plus écrasante lorsque rien, du moins le croyait-on jusque-là, ne faisait penser à une telle éventualité.

Quoi qu'il en soit, dans cet état de confusion, d'anesthésie et d'abattement survient alors la grande période des interrogations, la période des «si »: «si j'avais fait ça, si je n'avais pas fait ça, si j'avais vu, si j'avais pensé!». Et on essaie de refaire l'histoire à coups d'interprétations rétrospectives. On veut donner du sens et de l'importance à certains mots, à certains indices qui n'avaient évidemment ni la même valeur ni le même sens avant le suicide auquel on ne pensait peut-être même pas.

Nous essayons de développer notre empathie, notre sympathie, notre compassion - bien loin de la pitié - pour les familles frappées par un suicide inattendu, sans signe prémonitoire chez un proche qui ne semblait pas avoir de difficultés particulières et faisait même éventuellement profession de boute-en-train. Elles n'ont pas pu penser à une telle éventualité, ni donc s'y préparer ou essayer d'agir pour l'éviter. Mais nous pensons aussi avec attention et autant de compréhension aux familles qui vivent avec un déprimé chronique ou récurrent, comme dans certaines formes de la maladie maniaco-dépressive, où le suicide est toujours en perspective avec la pensée que la moindre erreur, la moindre défaillance, la moindre faiblesse risque d'être dramatiquement sanctionnée alors que l'intéressé le plus souvent ne sait même pas lui-même ce qui pourrait lui faire du bien. Au terme de plusieurs années de lutte contre la maladie et la mort, d'accompagnement épuisant et incertain, les proches peuvent en venir à souhaiter la fin de leur calvaire qui sera aussi la fin de celui de leur proche. Voilà une pensée qui n'est pas facilement acceptable consciemment. Elle peut le devenir lorsque la personne en deuil a profondément le sentiment que le suicidé a enfin trouvé la paix après des années de souffrance trop lourde. Le soulagement de l'être aimé en souffrance est acceptable - et c'est même un élément moteur du deuil après suicide -, celui d'en être enfin débarrassé l'est beaucoup moins facilement. Ce risque d'être une pensée bien culpabilisante.

La mort, au plus profond de nous, reste toujours peu ou prou acceptable. Lors d'un suicide, nous voudrions comprendre mais nous n'y arrivons pas dans la mesure où nous ne pouvons pas accepter non seulement la mort, ce que nous savons être déjà difficile, mais, en plus, le fait que l'être aimé de nous se soit ôté la vie, se soit donné la mort. Ces interrogations torturantes cesseront lorsque, au terme du long et pénible cheminement du travail de deuil, nous serons arrivés à voir diminuer notre colère, notre révolte, lorsque nous commencerons à accepter l'idée que l'être aimé avait des raisons, ses raisons à lui, qui ne sont pas les nôtres, et que nous commençons à penser que, peut-être, nous pourrions ne pas le connaître vraiment. Nous sommes alors sur la voie de reconnaître, avant d'accepter, le mystère que comporte toujours l'acte suicidaire. J'entends bien qu'il ne s'agit pas d'un mystère en soi; le côté mystérieux étant que nous ne pourrons jamais comprendre vraiment celui qui se suicide au moment de son acte, parce que nous ne pouvons pas être à sa place. Et peut-être que, assez souvent, lui-même n'a pas vraiment conscience des forces qui le poussent à cette extrémité. Car il faut aussi avoir raison de cette expression fallacieuse où le suicide apparaîtrait comme un choix. Hors des suicides oblatifs, par exemple des kamikazes ou de l'intifada ou de certains suicides pour échapper à d'autres maux extrêmes, le désespoir si profond qu'il conduit à la mort n'est pas un choix; il devient une force contraignante, une nécessité pour le faire cesser.

Cette pensée souvent apaisante pour les proches qui ont vécu au côté d'un grand déprimé suicidaire, cette pensée qu'il a enfin trouvé le soulagement et la paix est, au contraire, torturante pour ceux qui vivent un suicide imprévu, les faisant souffrir de cette douleur supposée qu'ils croient n'avoir pas su voir, le sentiment d'être passés à côté d'un élément essentiel de celui qu'ils aimaient, de l'avoir largement méconnu, d'être passé sans voir, sans comprendre, une ombre terrible sur leur amour. Accepter de reconnaître l'inconnu de l'autre - et surtout dans ces circonstances si tragiques -, c'est accepter de reconnaître que nous n'étions pas tout pour lui, pensée facile sur le plan conscient, pensée très difficile au plus profond.

\section{UNE MORT MYSTÉRIEUSE}

Pour tenter de mieux comprendre le deuil après suicide, interrogeons-nous quelques instants sur les motivations de l'acte suicidaire, étant bien conscients qu'il gardera toujours sa part de mystère et ne pourra manquer de nous laisser dans l'incertitude. Acte de la dernière violence dont les conséquences sont irréversibles, 
le suicide nous oblige à penser en premier lieu à l'être qui se l'inflige. Violence d'abord contre son corps... Non seulement il a perdu la vie mais il peut être plus ou moins mutilé, défiguré, ce qui ajoute encore à la souffrance des proches, leur imposant des images traumatiques plus pénibles. Ici la thanatopraxie montre son importance. Mais violence aussi contre son âme qui restera toujours marquée du sceau de la souffrance et du désespoir. Même s'il est parfois difficile aux intéressés de le reconnaître, cette violence est aussi dirigée contre les autres. Tous les proches - et le cercle des proches peut être très large - tous les proches sont déchirés, blessés, violentés par cet acte cruel à la fois contre soi-même et contre eux où s'expriment violence et haine secrète.

Si l'acte suicide exprime la réussite de la mort, il signe bien l'échec de la communication! Ce qui est agi brusquement, brutalement, impulsivement est bien ce qui n'a pas pu être dit, être partagé avec les autres. Dans ces suicides impulsifs, comme dans tous les actes impulsifs, il existe sans doute un défaut du travail psychique de mentalisation, un défaut de représentations: ce qui n'arrive pas à être éprouvé affectivement, à être pensé en représentations dans des mots ou par des images se déverse brusquement dans le comportement, le corps qui aurait pu être une autre voie de décharge n'a pas eu le temps de se rendre malade.

Le suicide est violence partagée, violence contre soi-même et violence contre les autres. L'acte suicide installe cette violence partagée dans la durée; le suicide est une inscription indélébile, un message. Ce qui n'a pas pu se dire dans l'amour est maintenant inscrit durablement dans la haine; c'est là le côté dramatique et malheureux du suicide. Meurtre, le suicide est un acte de sadisme.

À l'évidence, le suicidé s'en prend d'abord à lui-même. Il met fin à sa vie en détruisant son corps. Certains suicidaires ont peur de la mort et donnent le sentiment de s'y précipiter pour mieux y échapper tout comme d'autres préfèrent mourir que d'affronter la perspective de la vieillesse. Les manières de se tuer sont diverses mais les plus violentes, les armes à feu, la pendaison et plus rarement la chute d'un lieu élevé, sont les moyens les plus choisis. Ce message de violence, chaque suicidé l'exprime à sa manière en fonction des vicissitudes de son histoire et de sa vie intérieure.

C'est plutôt le moment de se rappeler que Freud comprenait le suicide comme un retournement contre soi de son propre sadisme:

[U]n névrosé n'éprouve pas d'intention suicidaire qui ne soit le résultat du retournement sur soi d'une impulsion meurtrière contre autrui.

[...]

[L]e moi ne peut se tuer que lorsqu'il peut, de par le retour de l'investissement d'objet, se traiter lui-même comme un objet, lorsqu'il lui est loisible de diriger contre lui-même l'hostilité qui vise un objet (Freud, 1920).

Le retour de l'investissement d'objet dont il est ici question est une situation de deuil, une situation de deuil narcissique, c'est-à-dire une perte au niveau de l'estime de soi, de ses idéaux, de ses illusions, de ce qui est attendu de l'objet bien plus qu'une perte au niveau de l'existence même de l'objet. Cette situation de deuil narcissique est la circonstance déclenchant le retournement contre le moi de son propre sadisme. Celui-ci, à un tel degré d'intensité, est l'expression privilégiée d'une ambivalence excessive qui n'a pas pu être relativisée, être en bonne partie maîtrisée comme il est habituel au cours du développement.

\section{C'EST POUR TENTER D'ÉCHAPPER À CE SENTIMENT} TORTURANT D'IMPUISSANCE QUE LES SURVIVANTS S'EFFORCENT D'ÉCLAIRER CE MYSTÈRE DU SUICIDE, DE CE SUICIDE, PAR UNE RECHERCHE FORCENÉE DE SENS.

Les situations extrêmes de douleur morale comme les tensions excessives, le deuil, la maladie et certaines périodes de changement dans le cours de la vie, l'adolescence et le vieillissement, plongent le sujet qui les éprouve dans la passivité et le font régresser. Le sadisme, anal de meurtre, oral de dévoration, est un des modes privilégiés de cette régression - l'identification narcissique en est un autre - et en même temps une ouverture directe sur l'activité. Mais il n'y a plus ici qu'un seul objet à sadiser à l'extrême: c'est soi-même! Et nous rendons alors raison à Freud «seul le sadisme vient résoudre l'énigme de la tendance au suicide» (Freud, 1920) et je pense aussi à ceux qui se tuent pour protéger les autres, en particulier de leur propre sadisme.

La violence contre les autres, ceux en particulier qui sont les plus proches et les plus aimés - de manière ambivalente, il est vrai -, est toujours présente dans l'acte suicide. Mais elle s'y inscrit de manière paradoxale: d'un côté l'objet d'amour est ainsi protégé de la violence meurtrière dont il était l'objet à l'intérieur du suici- dant, il n'est pas tué - le suicide réalisant un meurtre par procuration - mais de l'autre côté il est durablement, presque indélébilement marqué par la souffrance que cet acte de violence détermine à l'intérieur de lui (Hanus, 2004).

Le suicide est le triomphe ultime et pathétique de l'ambivalence. Dans le même acte où l'être se détruit en victime à l'image idéalisée de son objet d'amour, il veut le tuer à travers lui pour ne jamais le perdre. Nous devons alors faire l'hypothèse que les suicidaires, les suicidants et les suicidés n'ont pas pu entrer dans l'œuvre essentielle $\mathrm{du}$ "deuil narcissique», ils n'ont pas pu accepter / intégrer les séparations nécessaires à leur autonomisation pas plus que la dés-idéalisation de leurs premiers objets auxquels ils vouent idolâtrie et rancune jusqu'à s'en faire mourir à l'occasion d'une ultime déception narcissique.

Cette violence que le suicidé imprime dans le cœur de celui qui l'aime prend essentiellement la forme de sentiments de culpabilité torturants. En se demandant de manière obsédante ce qu'on n'a pas fait, ce qu'on n'a pas bien fait, ce qu'on a mal fait, on entre dans la question du sens. Et là encore l'endeuillé par suicide se trouve dans une situation paradoxale: il ne comprend pas, il lui faut absolument essayer de comprendre et il sait qu'il ne pourra jamais vraiment comprendre, quelque chose va toujours lui échapper. Son chemin d'évolution va passer par la nécessité d'accepter son impuissance, ses limites et l'amènera à reconnaître enfin à celui qui s'est tué la possibilité de disposer de lui-même.

La relation qui unit telle personne à telle autre est singulière, unique, particulière; elle a ses dimensions visibles et ses implications intérieures, invisibles et dont un certain nombre sont inconscientes. Chaque deuil est particulier et chaque suicide l'est également. La mort, le deuil et le suicide encore plus augmentent cette part de mystère, d'insaisissable, d'incompréhensible dans nos relations même les plus chères du fait que plus aucun échange n'est désormais possible.

Entre les deux pôles extrêmes du suicide impulsion perpétré en quelques secondes et le suicide ultime aboutissement au terme de difficultés psychiques insurmontables, 
la part du mystère dans le suicide est variable. Elle paraît souvent éclairée par les derniers mots du suicidé, la lettre ou les écrits qu'il a pu laisser. Ces messages vont être interprétés et ré-interprétés à l'infini par les survivants qui vont y chercher et essayer d'y trouver un sens, des sens. Mais dans sa lettre d'adieu le suicidant ne dit que ce qu'il veut bien dire, il ne dit qu'une partie de ce qu'il pense au moment où il l'écrit. Et il ne sait pas toujours luimême ce qui le pousse à agir ainsi. Le plus souvent, il parle de ses souffrances, de son désespoir et demande pardon à ceux qui restent.

La souffrance a déjà sa part de mystère dans le fait même qu'elle n'a pas pu être apaisée dans le partage. Le désespoir est lui aussi énigmatique qui résulte d'une perte totale de confiance dans ses propres possibilités d'évolution, de changement, de soulagement et dans les capacités des autres à y aider. C'est souvent ce constat d'impuissance qui, avec les sentiments de culpabilité, rend si douloureux le deuil après suicide. C'est pour tenter d'échapper à ce sentiment torturant d'impuissance que les survivants s'efforcent d'éclairer ce mystère du suicide, de ce suicide, par une recherche forcenée de sens. Et ainsi chaque endeuillé après suicide se redit que le suicide, au moment où il se réalise, est l'aboutissement de la conjonction qualitativement et quantitativement variée de plusieurs facteurs: l'agressivité et la violence, physique contre soi-même, morale contre autrui, le besoin d'échapper à des sentiments d'impuissance et de désespoir devenus insupportables, le besoin de mettre dans un acte ultime ce qui n'a jamais pu se dire avec des mots ni même s'éprouver dans des émotions discernables parce que restées trop confuses du fait de leur intensité explosive, le désir d'échapper à des dangers non maîtrisables et de s'offrir en sacrifice pour tenter de sauvegarder des valeurs jugées inestimables. Tous ces facteurs - et il peut en exister d'autres - ont une grande variété d'harmoniques.

Pour avancer dans son deuil, il va falloir accepter avec le temps de ne pas pouvoir tout comprendre, accepter cette part de mystère et, de ce fait, reconnâ̂tre encore une fois son impuissance. Que l'on considère surtout le suicide, ce suicide, comme une fuite ou plutôt comme l'apaisement inévitable d'une souffrance devenue insupportable, le suicidé dans son acte, par son acte, a déclaré forfait: il n'en peut plus! Nous avons déjà remarqué comment il transmet sa souffrance à ceux qui lui survivent. Il a souffert jusqu'à la limite de ses forces: maintenant c'est à eux de souffrir; c'est à eux de continuer de souffrir car ils souffraient déjà au contact du suicidaire dans l'incertitude de l'avenir (Fauré, 2007).

Mais derrière la souffrance tout se passe comme si le suicidé qui vient de déclarer forfait face aux conflits qui l'habitaient les transmettait aux survivants qui doivent maintenant y faire face. Ils doivent alors s'atteler à ce qu'il n'a pas réussi dans une continuité, un héritage, une fidélité. Ce message, ce travail transmis, est essentiellement d'arriver à dépasser l'ambivalence qui régnait dans les relations antérieures, d'accepter de souffrir le sadisme de celui qui a fait souffrir ses proches par sa disparition brutale. Il s'agit, en quelque sorte, d'accepter sa colère, sa révolte et son ressentiment vis-à-vis de celui qui se permet ainsi une telle offense, tout en lui pardonnant. La souffrance partagée et le pardon sont les grands remèdes aux sentiments de culpabilité.

Le suicide comporte parfois une dimension de sacrifice. C'est Ian Palach qui s'immole par le feu sur la place Wenceslas à Prague. Mais, de manière plus discrète, certains suicides, plus proches de nous, font appel à une telle motivation. Je l'ai constaté chez certains de mes malades qui pensent parfois que leur famille, leurs enfants seraient mieux sans eux. Il s'agit là bien sûr d'une idée dépressive mais qui est parfois d'un certain poids dans la détermination suicidaire. Certaines personnes refusent de prendre en considération la dimension de désespoir du suicide lorsqu'elle n'est pas criante; elles tiennent à conserver la possibilité d'un suicide philosophique comme un choix existentiel. Les équipes de soins palliatifs ont souvent constaté que les personnes qui demandent l'euthanasie sont en fait à la recherche de davantage de présence, d'accompagnement et de soulagement.

Quelques jeunes qui se sont suicidés ont laissé un texte où ils débattent, froidement en apparence, des avantages et surtout des inconvénients de la vie, s'efforcent de trouver des arguments en faveur de son inanité, de son absurdité. Ils se terminent souvent par «je n'ai pas demandé à vivre, je peux au moins avoir la liberté de mourir ». Derrière cette pesanteur de la vie, ce manque de liberté, nous sentons la souffrance et le désespoir.

\section{LES PARTICULARITÉS DU DEUIL APRÈS SUICIDE}

S'il est tout à fait vain de vouloir comparer la gravité des différents deuils, car celui que nous vivons présentement est toujours pour nous le plus difficile, les deuils après suicide sont cependant particulièrement lourds.

Le suicide ouvre un deuil traumatique; c'est une violence, c'est un meurtre.
Comme dans tous les deuils traumatiques, le travail de deuil ne pourra commencer que lorsque les images traumatiques de l'événement - qu'on ait été témoin ou qu'on en ait reçu le récit - auront pu être suffisamment exprimées avec toutes les émotions fortes qui s'y attachent. Tant que ce premier pas n'est pas fait, le travail de deuil ne peut pas se mettre en route. À chaque fois que l'endeuillé pense à celui qui est mort, il est de nouveau envahi par ces images traumatiques qui lui bloquent l'accès à tout autre souvenir. Ce besoin d'expression et de partage peut tarder à s'exprimer en raison du mouvement d'abattement, d'écrasement et d'anesthésie affective relative qui se manifeste assez souvent à l'orée de ces deuils.

La mise en route du travail de deuil est également plus difficile lorsque le suicide est survenu de manière tout à fait inattendue, imprévisible; le choc est alors encore plus rude. Des signes prémonitoires comme des difficultés dépressives, une tentative de suicide ou des menaces de suicide permettent, dès avant, au pré-deuil de se mettre en place. Si la mort par suicide doit survenir, cette éventualité est déjà venue à la pensée. Il n'y a rien de semblable en cas de suicide brutal sans sommation et c'est d'autant plus difficile.

L'acceptation de la réalité matérielle du suicide ne fait, en général, guère problème. Mais il y a des exceptions lorsque la situation peut prêter à confusion entre un accident et un suicide, entre un suicide ou un acte de malveillance. Mais la réalité psychique est, elle, beaucoup plus difficile à accepter, en particulier toutes les émotions fortes de colère et de ressentiment à l'égard du suicidé. La colère est présente dans tous les deuils où l'on se sent abandonné par celui qui vient de mourir. Elle est encore plus forte après le suicide puisque c'est de son propre fait que cette personne nous a quittés. Mais ces sentiments sont difficiles à reconnaître puis à accepter car ils risquent d'augmenter encore l'importance, l'intensité et la profondeur des sentiments de culpabilité des endeuillés qui sont déjà bien assez pesants.

Le travail d'intériorisation de la relation est lui aussi rendu plus difficile non seulement en raison de la présence des images traumatiques toujours prêtes à se sur-imprimer aux souvenirs dont l'évocation est nécessaire au travail de deuil mais aussi en raison du caractère très ambivalent de la relation qui préexistait au suicide. Les identifications générales et nécessaires dans tout travail de deuil sont également problématiques après le suicide. Il faut tout un cheminement à travers la souffrance partagée et le pardon, afin de surmonter une partie de l'ambivalence, 
avant qu'il ne soit possible de redécouvrir vraiment tous les aspects positifs de la personne disparue par suicide à laquelle il est alors possible de s'identifier sur certains points.

Mais c'est bien sûr au niveau des sentiments de culpabilité que le travail de deuil après un suicide est bien plus difficile et souvent compliqué. Les sentiments de culpabilité inconscients en relation avec l'ambivalence présente dans toute relation ne manquent dans aucun travail de deuil et rendent compte en partie de sa longueur, de sa difficulté et de son caractère douloureux. Ils existent également dans ces deuils après suicide, mais ils sont renforcés par des sentiments conscients de culpabilité du fait de l'impuissance où l'on s'est finalement trouvé à pouvoir aider efficacement l'être aimé qui a fini par se supprimer.

Le suicide est donc un facteur de risque dans le deuil. De fait, on retrouve un plus grand nombre de suicides chez les endeuillés après suicide. Cette apparente contagiosité du suicide est à mettre sur le compte de la conjonction entre l'identification ambivalente au suicidé et la poussée de puissants sentiments de culpabilité: se suicider à son tour pour le rejoindre et se punir!

Dans ces conditions les endeuillés après suicide doivent faire l'objet d'une attention particulière, encore redoublée lorsqu'il s'agit de personnes à risque comme les enfants, les adolescents, les vieillards, les personnes solitaires et fragiles physiquement, moralement ou socialement. En plus de l'accompagnement naturel de proximité par l'entourage, un suivi sera mis en place pour offrir un espace d'échanges de paroles et d'émotions en faisant appel aux intervenants professionnels, soignants ou travailleurs sociaux et aux structures associatives qualifiées.

Les groupes de soutien pour les personnes endeuillées après suicide mis en place en milieu associatif depuis plusieurs années ont montré leur intérêt et ils ont tendance actuellement à se développer en Europe, en particulier au sein de la Fédération européenne Vivre son deuil. Nous savons bien qu'ils sont largement plus répandus et depuis plus longtemps au Canada et particulièrement au Québec ${ }^{1}$. Si l'animation des groupes de paroles pour les personnes en deuil demandent des compétences, celle des groupes d'endeuillés après suicide en exigent de particulières. Car, s'il n'est malheureusement pas possible de prévenir efficacement tous les suicides et toutes les tentatives, il serait particulièrement malheureux qu'ils surviennent chez un endeuillé qui fréquente un tel groupe qui s'en trouverait, de ce fait, très probablement fortement critiqué.
On ne saurait trop insister sur le fait que les enfants dont un des parents s'est suicidé ont besoin, pour faire face à leur deuil et continuer leur développement, d'une attention toute particulière qui commence par la nécessité de ne pas leur cacher la vérité même si on doit la leur faire connaître avec doigté et progressivement. Pour les accompagner efficacement ainsi que les autres membres de la famille, il est nécessaire de savoir, d'une part, que l'enfant se sent toujours coupable de la mort d'un de ses proches et, d'autre part, qu'il ne peut pas comprendre, à ce stade de son développement, qu'une personne qui l'aime puisse se supprimer: sa culpabilité s'en trouve redoublée. Elle est encore renforcée lorsque l'entourage n'est pas en état de reconnaître la réalité du suicide. Ces enfants endeuillés trouvent leur place dans les groupes d'enfants en deuil que nous avons mis en place depuis plusieurs années dans nos associations.

$$
* * *
$$

Après un cheminement long, lent, pénible et douloureux, beaucoup d'endeuillés après suicide en viennent à s'intéresser à la prévention du suicide qui est toujours une préoccupation majeure pour les travailleurs sociaux et tous ceux qui s'intéressent - soignants et non-soignants - à la santé publique ${ }^{2}$.

Le suicide est une tentation naturelle pour toutes les personnes en deuil, surtout lorsqu'il s'agit d'un deuil important - l'importance d'un deuil est quelque chose de tout à fait subjectif - chez une personne à risque. Proposer de l'aide aux endeuillés en difficultés, offrir de l'aide aux enfants endeuillés est déjà un premier grand pas dans la prévention.

Prendre en compte toutes les tentatives de suicide même lorsque le pronostic vital ne paraît pas gravement engagé comme un cri d'appel d'une souffrance qui a besoin d'être davantage reconnue, en s'efforçant de se tenir à égale distance de la banalisation et de la dramatisation. C'est l'occasion de mettre en place une prise en charge assez souple mais assez régulière à la fois du suicidant, souvent un jeune, et de son entourage familial en s'efforçant surtout de rétablir la communication et l'échange entre les uns et les autres.

Assurer un meilleur dépistage, un meilleur diagnostic et un meilleur traitement des états dépressifs dont un bon nombre sont encore méconnus. Les états dépressifs se soignent et presque tous guérissent; encore faut-il consulter et prendre correctement son traitement. Mais bien en amont, il est nécessaire de faire prendre conscience au grand public que la dépression n'est pas une honte, qu'elle doit être reconnue, prise en compte et soignée. Ce qui nécessite également la formation des médecins et des intervenants sociaux qui doivent favoriser le dépistage des dépressions et l'orientation des déprimés.

Porter attention à toute forme de solitude, de désarroi, de dénuement en s'efforçant de rétablir pour toutes ces personnes des liens de contacts et de communications. Il s'agit bien de l'accompagnement des personnes en solitude et en difficulté comme le réalisent, en particulier chez nous, les bénévoles des petits frères des Pauvres auprès des personnes âgées.

Enfin la prévention du suicide et des tentatives de suicide nécessite déjà que l'on prête une grande attention aux plus jeunes, les enfants et les adolescents, à tous ceux qui sont en difficulté en particulier du fait de la désunion familiale ou des difficultés relationnelles ou sociales qui émergent, du fait qu'ils ont été précocement confrontés à des deuils et des séparations ou à des fragilités de santé physique ou psychologique. Surtout lorsque les familles ne sont plus en état d'être le milieu naturel de protection des enfants, c'est alors à tous les intervenants qui sont en contact avec eux de se montrer vigilants à tout changement notable dans leur caractère ou leur comportement car c'est à ce niveau que s'exprime la souffrance dépressive des plus jeunes. C'est en entendant cette souffrance, en lui ménageant un espace d'expression et d'écoute que pourra être évitée, souhaitons-le, l'apparition du désespoir qui pousserait à l'extrême !

\section{Bibliographie}

FAURÉ, C. (2007). Après le suicide d'un proche, Paris, Albin Michel.

FREUD, S. (1924). «Les résistances contre la psychanalyse », Euvres complètes, t. XVII, Paris, Presses universitaires de France.

FREUD, S. (1920). «Psychogénèse d'un cas d'homosexualité féminine", EEuvres complètes, t. XV, Paris, Presses universitaires de France.

FREUD, S. (1915a). «Actuelles sur la guerre et la mort », CEuvres complètes, t. XIII, Paris, Presses universitaires de France.

FREUD, S. (1915b). "Deuil et mélancolie», Euvres complètes, t. XIII, Paris, Presses universitaires de France.

HANUS, M. (2004). Le deuil après suicide, Paris, Maloine.

\section{Notes}

1. Nous avons pu en discuter largement au cours du premier Congrès international francophone de prévention du suicide qui s'est tenu à Québec en avril 2000.

2. C'est ainsi qu'a été créée en France, il y a quelques années, avec le soutien de la Direction générale de la santé, l'Union nationale pour la prévention du suicide (UNPS). 